\title{
SinkTrail Protocol Extention with Dead End Free Topology Used in WSN
}

\author{
Bhushan Zatkar ${ }^{1}$, Nilesh Vani \\ ${ }^{1}$ Student, Department of Computer Engineering, Godavari College of Engineering, Jalgaon, Maharashtra, India \\ ${ }^{2}$ Assistant Professor, Department of Computer Engineering, Godavari College of Engineering, Jalgaon, Maharashtra, India
}

\begin{abstract}
Wireless sensor network (WSN) uses a sensor node to observer and stored environmental physical condition and organised the data at central location. There are to much divercity in the movement of mobile sinks due to continueous changes in the terrestrial, without using GPS devices or predefined landmark. SinkTrail sends data packets by making a logical coordinate system using routing and forwardings, so that it can be used in wrost application scenario. A local maximum problem that is dead end while routing or forwarding data packetsis the primary drawback of sinktrail .In order to overcome from this primary drawback we extend sinkTrail with a Dead End Free Topology (DEFT) in our proposed system. Such system can have the capability and efficiency to deal with the dead end occurred in the sink trail protocol. This type of system also provide facility for do not occurred dead end situation and also find shortest routing path using dead end free topology.
\end{abstract}

Keywords: SinkTrail Protocol, Greedy Algorithm, Dead End Free Topology, Global Positioning System.

\section{Introduction}

The use of Wireless Sensor Networks (WSNs) has tremandously increased in real time application. For a wireless sensor network it's sensor node is very important. This sensor node are deployed in the geographical region for sensing a perticular condition like temprature, humidity etc with a very less human interaction. Each sensor node consist of individul batter for their own opeation so energy saving is a important factor for sensor network because this batterys have some limitation. Data collection recent research reveals that it allowing as well as leveraging sink mobility is more promising for energy efficient data gathering, rather than reporting data through long, multihop and error prone routes to a static sink using tree or cluster network structure. In the field animal or vehicles consider as a mobile sink they equipped with radio devices and send directly information to sensor. They provide a benefits for reduce energy consumption and shorter data transmission. Mobile sink used for data gathering is a new challenge for sensor network application. Data gathering is important used of sensor network. More challenging work for researchers is that minimize the time for data gathering. This is better benefit for sink mobility. Now some mobile element scheduling protocol has been proposed. Using control sink mobility they achieve efficient data collection. But for purpose of data collection dead end problem may be occurred. Same problem occurred in SinkTrail. SinkTrail solved a problem of movement prediction for data gathering with mobile sink. SinkTrail although uses shortest path for routing data. It may encounter dead end during its routing. Our proposed is to modify SinkTrail protocol to find an alternative path if dead end occurs. Using Dead End Free Topology (DEFT), this issue of dead end can be overcome in SinkTrail.

In this paper chapter 2 gives details about literature review, chapter 3 gives problem definition. Chapter 4 provides proposed solution to the problem definition. Chapter 5 provides graphical information related to simulation and result. Finally conclusion is given in chapter 6 .

\section{Literature Survey}

In [13] a Greedy Perimeter Stateless Routing (GPSR) is praposed which farwords a packet $t$ destination with a novel routing protocol for wireless datagram networks that uses the positions of routers. GPSR makes greedy forwarding decisions using only information about a router's immediate neighbors in the network topology. When a packet reaches a region where greedy forwarding is impossible, the algorithm recovers by routing around the perimeter of the region. By keeping state only about the local topology, GPSR scales better in per-router state than shortest-path and ad-hoc routing protocols as the number of network destinations increases.

Keally et al. for the purpose of data reporting the predict a sink location using Sequential Monte Carlo theory. Complexity of sinktrail is much lower using prediction techniques. For SinkTrail protocol inspiration Virtual coordinate routing is important [5].

Yong Liu et al. in [3] and Subhabrata Sen et al. proposed on for each sensor node in WSN the control of network have done using mobile sink movement to query. As well as for data gathering tour they intentionally minimize the length of each data for SinkTrail [4].

Chih-Hsun Anthony Chou,Kuo-Feng Ssu,Hewijin Christine Jiau,Wei-TongWang and Chao Wang et al. in [2] have proposed Existing topology maintenance protocols conserve energy by scheduling the network nodes to a sleep mode when a node is not currently involved in a communication activity. Based on the knowledge of the geographical locations of each of the nodes within the network, the GAF protocol divides the total network area into an arrangement of structured smaller grids such that each grid contains only one active node.

\section{Volume 4 Issue 12, December 2015}




\section{International Journal of Science and Research (IJSR) \\ ISSN (Online): 2319-7064}

Index Copernicus Value (2013): 6.14 | Impact Factor (2014): 5.611

\section{Problem Definition}

The Most of the Existing System uses Greedy Forwarding Approach. The Most Forward with fixed Radius (MFR) algorithm is widely used for next hop selection in geographic forwarding schemes [11]. In MFR, the current relay node always selects the neighbor closest to the destination as the next relay. However, when the current node cannot locate any neighbor closer to the destination than itself, the packet reaches a dead end. Several recovery strategies have been proposed for dealing with such an event. For example, in the scheme proposed by Finn in [13], the current relay node recursively searches its neighbors neighbors to find a node closer to the destination than itself. Woo and Singh proposed a scalable location update-based routing protocol in which the current relay node interrogates its neighbors for an alternative route to the destination [12]. Meanwhile, in GPSR [6], the current relay node first creates a planar subgraph using the relative neighborhood graph (RNG) [3] and then routes around the dead end in accordance with the right-hand rule. Various intermediate node forwarding techniques have also been proposed to resolve the dead end situation by forwarding the packet to specific positions within the network [8], [9]. However, Frey and Stojmenovic provided a formal proof that these schemes cannot guarantee packet delivery in specific graph classes or even any arbitrary planar graphs [4].

Disadvantages of Existing

1)Large data packets required more energy consumption and energy cost.

2)Could not work without GPS devices to dicover sensor node.

3)Buffer management also plays a significant role in achieving our goal.

4)Bandwidth has a very fine impact on the buffer management scheme. Based on the available bandwidth the dynamicity of the threshold is dependent, so we need an effective \& efficient bandwidth estimation technique. There is a traditional buffer management scheme RED (Random Early Detection) which works on threshold principle, but we are in the need to intelligently handle this threshold dynamically based on the available bandwidth.

5)Neighbourhood node selection problem.

Neighbourhood node selection problem occurred because of route and link selection. If we give a multiple path to each node then it have ability to select other node. For selection it first checks whether neighbour node available or not using node selection algorithm. Then checks whether neighbour node is active or in sleeping mode to the destination. After that they find selected node, it is near as compare to destination node. If its near to the destination node then checks charging capacity.Finnaly select it and forward packet to it. Loss of connection is a major issue in SinkTrail network. Sometime node is not a range or some internal problem occurs in node. At that time another route path is selected to a requested node. Then it make connection with then and perform operation as per its functionality. Packet Loss is a critical problem in SinkTrail protocol. Packet is a collection of data. It is very confidental.
In Wireless Sensor Network, packet loss is due to unavailability of sensor node. Some internal problem occurred in sensor node so its not working. Source node forward a packet to destination, but its not reach to a destination. Its working upto a battery power. If it active long time then its battery is discharged. If we give a facility to next node selection. So, such type of problem is not occurred and packet will be send from source node to destination node successfully. In this dissertation we focus our efforts on achieving following goals :

1) Solve a problem of neighborhood node selection.

2) Remove a loss connection condition in sensor node.

3) Remove packet losses and forward a packet to particular destination node.

4) Inactive a discharge node and remove it on a network also replace to another one.

5) Improve PDR and Throughput as compare to greedy forwarding.

All above problem can be solved using dead end free topology. As well as some in geographic forwarding poses major problem of dead end encounter. At that time Dead End Free Topology create some addition overhead and using construction operation via an alternative route they forward a packet to destination.

\section{Proposed Solution}

As per the problems discussed above the proposed protocols feature low-complexity and reduced control overheads. We propose an improvement to the SinkTrail. Basically it is a proactive data reporting protocol and self adaptive for various application scenario. For the purpose of data collection in SinkTrail, mobile sink continuously move in relatively slow speed in the field. It broadcasted control messages at certain point using lower frequency for existing data gathering. The mobile sink shows the footprint of the position. This footprint is nothing but a virtual landmark. Using this landmark sensor can easily identify the hop count distance. In distance the sensor nodes coordinate represent the combined logical distance coordinate space constructed by the mobile sink. The coordinate of mobile sink is nothing but its hop count distance from previous landmark to current location. At last problem of movement prediction for data gathering solve by using SinkTrail. In SinkTrail in place of greedy forwarding, if use a DEFT then its more beneficial for packet forwarding and data gathering.

As per our problem definition, here we explain Dead End Free Topology in detail. For deploying a wireless sensor network, energy consumption is fundamentally required.

The main role of various topology and control protocol is that turning off unnecessary node for energy conservation and maintain a constant level of routing fidelity. Same like that SinkTrail protocol commonly integrate with any routing scheme. whenever a packet encounters a dead end, additional overheads must be paid to forward the packet to the destination via an alternative route. 


\section{International Journal of Science and Research (IJSR) \\ ISSN (Online): 2319-7064}

Index Copernicus Value (2013): 6.14 | Impact Factor (2014): 5.611

\subsection{Working of DEFT}

In wireless sensor network a dead end situation may occur when the current node does not have any active neighbour node for forwording packet upto the destination and because of this the packet could not reachs to destination node. The working of DEFT slightly different, first an initial node is chosen randomly at prescribed periodic intervals and is then used as the starting point for a global topology construction process.

In constructing the topology, neighbouring node to the initial node are activated based on their ability to satisfy the local dead-end free condition.

The selected active neighbours then perform a similar activation procedure with their own neighbouring nodes. This construction process continue iteratively until all the active nodes satisfy the local dead-end free condition(LDF), and there for , by defination, the network satisfies the global dead-end free condition (GDF).

\subsubsection{Dead End Handling in Geographic Forwarding}

The Most Forward with fixed Radius (MFR) algorithm is widely used for next hop selection in geographic forwarding scheme. In MFR, the current relay node always select the neighbor closest to the destination as the next relay.

\subsubsection{Dead End Free Verification}

1)GDF Condition: The dead-end situation does not occur at any node in the network.

2)LDF Condition: If the transmission circle of the node is fully covered by the perpendicular bisectors with its neighbours, the node is dead-end free.

\subsubsection{Dead End Free Topology Construction}

Dead End Free Topology construction is divided into 7 state.

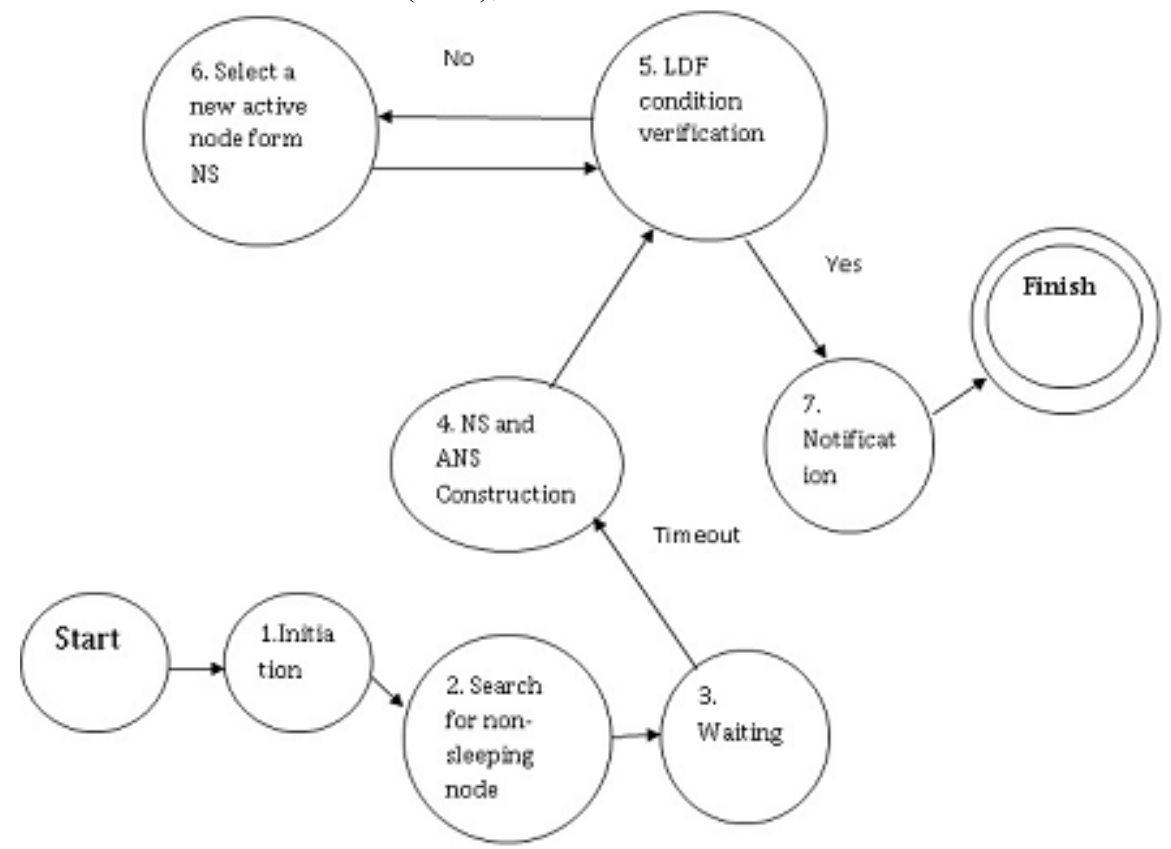

Figure 1: DEFT Construction Operation

Initially, all the nodes are to be taken undecided mode, i.e., the most appropriate mode for each node ie yet to be determined. The process commences when the sink randomly nominates a node to become an active node, hence causing the node to transit to state 1 . In state 1 , the selected node (referred to hereafter as the initiator) sets its mode to active and enters state 2. The initiator then broadcast a message to search for nonsleeping neighbors and then transmit to state 3 for receiving response. Once a nonsleeping node receive the message from the initiator, it reports its operation mode to the initiator. When the initiators timer expire, the process move to state 4 .

Now initiators first adds all the nodes which have replied to its broadcast to a neighbour set, then adds those nodes which have replied to its broadcast to a neighbour set (NS). Then adds those nodes which are in an active mode to an active neighbour set (ANS), and finally transmit to state 5. From state 5 two transition are possible, i.e. to state 6 or to state 7 , respectively. The process transmit to state 6 if the initiator fails to satisfy the LDF condition with the nodes in its current ANS: otherwise, it transmit to state 7(i.e., the initiator satisfies the LDF condition). In state 6, the initiator select a new active node from its NS using active node selection algorithm and then add this nodes to its ANS. The process then returns to state 5 to verify whether or not the updated ANS to satisfy LDF condition. In state 7 , the node which have been selected by the initiator to be active nodes are notify and they have been designed at new initiators. Each of this node then perform a topology construction process describe above using its own local neighbour. Meanwhile, those node within a voronoi polygon enclosed by the perpendicular bisector between the original initiator and the nodes in its ANS are notify to enter the sleep mode. The process then transmit to the end state i.e.,to Finish[2].

Advantages of proposed system :

1)It required less energy consumption.

\section{Volume 4 Issue 12, December 2015}




\section{International Journal of Science and Research (IJSR) \\ ISSN (Online): 2319-7064}

Index Copernicus Value (2013): 6.14 | Impact Factor (2014): 5.611

2)It's normally reduces an active node.

3)Provides higher rate and atio for packet forwarding.

4) Active node requirement is less as its find shortest path.

5)Provides high stimulating parameter as compare to existing forwarding technique.

On the basis of this rules and advantages they select nearest neighbour node for alternative path and gather data from destination node.

\section{Simulation and Result}

We perform experiment to evaluate the PDR and throughtput.
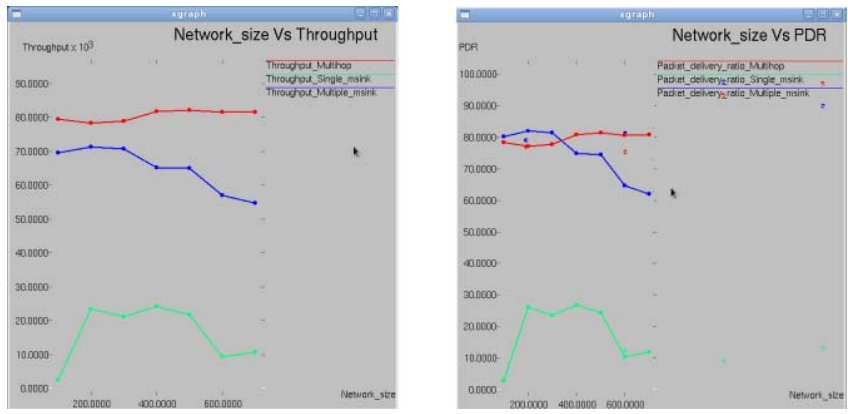

Figure 2 Dead End Greedy Forwarding

In Figure 2 Graph show greedy forwarding simulation result.
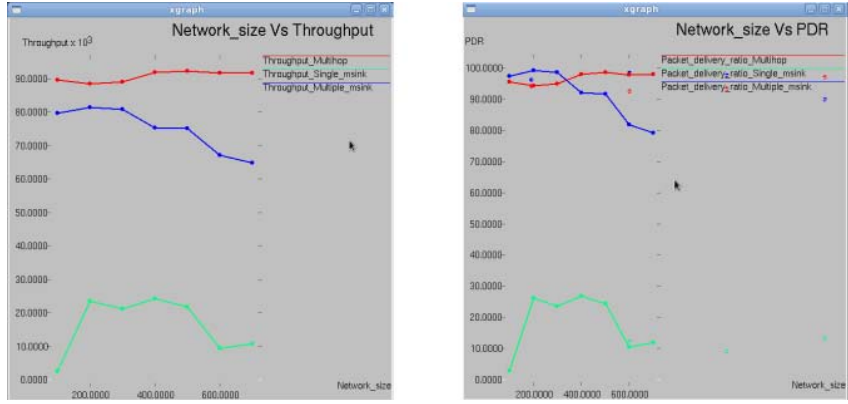

Figure 3 Dead End Free Topology

In Figure 3 Graph shows a dead end free topology simulation result. As it can be seen from the graph the proposed, archives the higher throughout and PDR ratio compared to the sinktrail with greedy approach. Finally we conclude that dead end free topology is more beneficial than greedy forwarding algorithm.

\section{Conclusion}

We proposed an extention to a SinkTrail with Dead End Free Topology. This wireless sensor protocol has different types of uses. But SinkTrail has a major drawback of dead end for data gathering. We combine dead end free topology maintenance protocol, designed as DEFT, for the construction of dead end free topologies for wireless sensor network using a minimum number of active nodes. DEFT is combines with any MFR-based geographic forwarding algorithm to achieves less energy consumption for each sensor node and to occur a minimum number of dead end event. The proposal may give the topology constructed by DEFT as dead end free in most simulated scenrios.
Furthermore, even when positional error exist, DEFT ensures the only a limited number of dead end event take place. Hence we extend SinkTrail Protocol with DEFT to solve the primary problem of dead end in our proposal.

\section{Acknowledgement}

We would like to take this opportunity to express our profound gratitude and deep regard to my Guide Prof. Nilesh Vani, for his exemplary guidance, valuable feedback and constant encouragement throughout the duration of the project. I would like to thanks our principal and the entire staff member who directly and indirectly guide me. Never the less I also like to thanks my parents and friends to help me during the completion of this task

\section{References}

[1] Jung, Keechul, Kwang In Kim, and Anil K Jain. "Text information extraction in images and video: a survey." Pattern recognition 37.5 (2004): 977-997.

[2] Wu, Victor, Raghavan Manmatha, and Edward $\mathrm{M}$. Riseman. "Textfinder: An automatic system to detect and recognize text in images." IEEE Transactions on pattern analysis and machine intelligence 21.11 (1999): 1224-1229.

[3] Eikvil, Line. "Optical Character Recognition." citeseer. ist. psu. edu/142042. html (1993).

[4] Liang, Su, M. Adviser-Ahmadi, and M. AdviserShridhar. "Restoration and segmentation of machine printed documents." (1996).

[5] Safronov, Kirill, Ing Igor Tchouchenkov, and Ing Heinz Wrn."Optical Character Recognition Using Optimisation Algorithms." Proceedings of the Ninth InternationalWorkshop on Computer Science and Information Technologies, Russia. 2007.

[6] Yanikoglu, Berrin A."Pitch-based segmentation and recognition of dot-matrix text." International Journal on Document Analysis and Recognition 3.1 (2000): 34-39.

[7] Grafmller, Martin, and Jrgen Beyerer."Segmentation of printed gray scale dot matrix characters." Proceedings of 14th world multi-conference on systemics, cybernetics and informatics WMSCI. Vol. 2. 2010.

[8] Zhang, Jing. "Extraction of Text Objects in Image and Video Documents". Diss. University of South Florida, 2012.

[9] Okun, Oleg G. "Morphological filter for text extraction from textured background." International Symposium on Optical Science and Technology. International Society for Optics and Photonics, 2001.

[10] Sachin, S. P., K. L. Banumathi, and R. Vanitha. "Database Development of Historical Documents: Skew Detection and Correction."

[11]Hull, Jonathan J. "Document image skew detection: Survey and annotated bibliography. Series in Machine Perception and Artificial Intelligence 29 (1998): 40-66.

[12] Papandreou, A., and B. Gatos. "A novel skew detection technique based on vertical projections." Proceedings of the International Conference on Document Analysis and Recognition, ICDAR. 2011. 384-388.

\section{Volume 4 Issue 12, December 2015}




\section{International Journal of Science and Research (IJSR) \\ ISSN (Online): 2319-7064}

Index Copernicus Value (2013): 6.14 | Impact Factor (2014): 5.611

[13]B. Karp and H.T. Kung, "GPSR: Greedy perimeter statless Routing for Wireless Networks," Proc. ACM Mobilcom, pp. 120-130, Aug.2000.

\section{Author Profile}

Bhushan Zatkar received his B.E. in Computers Engineering from Govt. College of Engg. Jalgaon in 2012-13. He is currently working as student in Godavari College of engg. Jalgaon for his M.E. His area of interest is networking, Software testing. 